\title{
ÜBERSICHT
}

\section{„Psychopathy“ im Kindes- und Jugendalter}

\section{Ein Update: Erkenntnisse und Perspektiven}

\author{
Nora Ortner $^{2}$ ID $\cdot$ Maximilian Preiß ${ }^{2} \cdot$ Kathrin Sevecke ${ }^{1,2}$ \\ Eingegangen: 4. Juni 2018 / Angenommen: 7. Juni 2018 / Online publiziert: 2. Juli 2018 \\ (c) Der/die Autor(en) 2018
}

\section{Zusammenfassung}

Psychopathy beschreibt eine schwere Persönlichkeitspathologie, welche sich durch Extremformen von aggressiv-antisozialem und kriminellem Verhalten sowie schwerwiegenden affektiven und interpersonalen Defiziten kennzeichnet. In der Erforschung der Psychopathy wird der Fokus zunehmend auf das Auftreten und Erkennen dieser Pathologien im Kindesund Jugendalter sowie deren Verlauf und Stabilität in Heranwachsenden gelegt. Hierbei finden sich eindeutige Hinweise darauf, dass Persönlichkeitsdimensionen der Psychopathy bereits im Kindes- und Jugendalter auftreten können, was eine Früherkennung durch altersspezifische Untersuchungsinstrumente ermöglicht. Aufgrund des deutlichen Zusammenhangs zwischen Psychopathy und schwerer, wiederholter und großteils behandlungsresistenter Gewalttätigkeit und den daraus entstehenden öffentlichen Schäden soll die Forschung mit Kindern und Jugendlichen dazu dienen, das Konstrukt der Psychopathy noch klarer zu definieren und aus einem besseren Verständnis der zugrunde liegenden Pathologien spezifischere Typologien sowie effektive Behandlungsstrategien zu entwickeln. Hierfür ist es von besonderer Bedeutung, die dimensionalen Faktoren der Psychopathy, wie sie auch bei Kindern und Adoleszenten auftreten, in ihrer Heterogenität zu erfassen und auf ihre Stabilität im Entwicklungsverlauf zu überprüfen.

Schlüsselwörter Delinquenz $\cdot$ Aggressives Verhalten $\cdot$ Psychopathy $\cdot$ Adoleszenz $\cdot$ Persönlichkeitspathologie

Prim. Univ.-Prof. Dr. Kathrin Sevecke

kathrin.sevecke@tirol-kliniken.at

1 Univ. Klinik für Psychiatrie, Psychotherapie und Psychosomatik im Kindes- und Jugendalter, Anichstraße 35, 6020 Innsbruck, Österreich

2 Abteilung für Psychiatrie, Psychotherapie und Psychosomatik im Kindes- und Jugendalter A.ö. Landeskrankenhaus Hall, Milser Straße 10, 6060 Hall in Tirol, Österreich 


\title{
Psychopathy in childhood and adolescence
}

An update: knowledge and perspectives

\begin{abstract}
The term psychopathy is defined as a severe personality pathology, which is characterized by extreme aggressive, antisocial and violent behavior as well as affective and interpersonal deficits. In recent years, research into the construct of psychopathy has often focused on the incidence and detection of such pathologies in children and adolescents. In this context, a point of interest is to explore the course and stability of psychopathic personality dimensions throughout youth. Results demonstrate clear evidence for the occurrence of personality traits characteristic for psychopathy in early childhood and adulthood, which facilitates early detection with the aid of age-specific diagnostic instruments.

The distinct correlation between psychopathy and severe, repeated and largely treatment refractory violence, which results in substantial public damage, calls for further research in children and adolescents in order to define the construct of psychopathy more precisely, to further illuminate underlying pathologies and consequently develop more specific typologies and effective treatment strategies. To that end, it is of particular importance to correctly capture dimensional factors of psychopathy in youth and to examine their stability throughout stages of adolescent development.
\end{abstract}

Keywords Delinquency $\cdot$ Aggressive Behavior $\cdot$ Psychopathy $\cdot$ Adolescence $\cdot$ Personality pathology

\section{Begriffsdefinition}

Die Konzeptualisierung der Psychopathy nach heutigem Verständnis beginnt mit der Definition nach Cleckley, welcher erstmals in den 1940er-Jahren den Begriff der „mask of sanity“ prägte. Dieser beschreibt eine vordergründig gesunde Fassade in Patienten mit dahinterliegenden, schwerwiegenden Persönlichkeitspathologien, welche sich in rücksichtslosem, waghalsigem und empathielosem Verhalten niederschlagen (Cleckley 1941). Dieses erste Modell der Psychopathy diente u. a. als Abgrenzung zu Erkrankungen mit psychotischen Symptomen, indem es den kohärenten Denkstil und das - außerhalb von Gewalttaten bestehende - gute soziale Funktionsniveau dieser Patientengruppe hervorhob. Bereits vor Cleckleys umfassender Konzeptualisierung wurde diese Subgruppe von Gewalttätern etwa von Kraepelin als „Schwindler“ beschrieben - die Betroffenen kennzeichnen sich dementsprechend einerseits durch fehlende Empathie, hohe Gewaltbereitschaft und fehlende Scham- oder Reuegefühle, kompensieren diese jedoch durch Anpassungsmechanismen wie manipulative und scheinbar sozialkompetente Verhaltensweisen (Patrick 2018).

In der Weiterentwicklung der ersten Definition nach Cleckley verweisen spätere Modelle auf eine mangelhafte Erkennung bzw. verminderte Reaktion auf schmerz- oder angstauslösende Reize, was als ein Faktor in massiv antisozialen Verhaltensweisen in Psychopathy-Populationen gewertet wird (Lykken 1957).

Nach diesen zunächst rein klinischen Beschreibungen versuchte erstmals Hare im Jahre 1991 mit der Psychopathy Checklist - Revised (PCL-R) und einem Zwei-FaktorenModell einerseits Kernfaktoren der Psychopathy so akkurat und differenziert wie möglich zu erfassen und andererseits ein Messinstrument zu erschaffen, um diese Faktoren zu operationalisieren und empirisch fassbar zu machen (Harpur et al. 1989; Hare et al. 1991).

Etwas später konzipierten Cooke und Michie hierauf aufbauend ein Drei-Faktoren-Modell (Cooke und Michie 2001). Wesentliche Veränderungen waren die Aufspaltung eines zuvor zusammengeschlossenen Faktors in einen interpersonellen (,,arrogant, betrügerisch, zwischenmenschlich“) und einen affektiven (,defizitär affektive Erfahrung“) Faktor; untergeordnete Items blieben dabei weitestgehend unverändert. Als dritten Faktor, legten sie - wie Hare - „impulsives unverantwortliches Verhalten“ mit dazugehörigen Items fest, klammerten jedoch antisoziale Charaktereigenschaften mit der Argumentation aus, dass antisoziales Verhalten lediglich eine kausale Folge und nicht Ursache für Psychopathy darstelle (Cooke et al. 2012).

Kurz darauf überarbeitete Hare die PCL-R, übernahm weitestgehend die Veränderungen von Cooke und Michie und fügte die Antisozialität unter Zurückweisung ihrer Argumentation als vierten Faktor wieder ein (Hare 2003).

Schlussendlich beschreibt das aktuelle Konzept der Psychopathy nach Hare eine schwere Persönlichkeitsstörung, welche sich durch vier dimensionale Faktoren erfassen lässt: 1) interpersonaler Faktor, 2) affektiver Faktor, 3) Lifestyle-Faktor, 4) antisozialer Faktor (s. Tab. 1). Die ersten beiden Faktoren dienen hierbei zur Erfassung von pathologischen Persönlichkeitsmerkmalen wie erheblich übersteigertem Selbstwertgefühl, Manipulation und Selbstdarstellung einerseits sowie mangelndem Gewissen, Gefühlskälte und fehlender Empathie andererseits. Der Lifestyle-Faktor beinhaltet Stimulationsbedürfnis, parasitäre Orientierung, Fehlen von Zielen sowie Impulsivität und Verantwortungslosigkeit, während der antisoziale Faktor zur Erfassung abweichender, aggressiv-krimineller 
Tab. 1 Psychopathy-Konstrukt nach Hare. (Modifiziert nach Sevecke und Krischer 2014)

\begin{tabular}{|c|c|c|c|}
\hline \multirow{2}{*}{$\begin{array}{l}\text { Kerndimensionen } \\
\text { Interpersonaler Faktor }\end{array}$} & \multirow[b]{2}{*}{ Affektiver Faktor } & \multicolumn{2}{|l|}{ Verhaltensdimensionen } \\
\hline & & Lifestyle-Faktor & Antisozialer Faktor \\
\hline Selbstdarstellung & Mangel an Gewissensbissen & $\begin{array}{l}\text { Stimulationsbedürfnis } \\
\text { Parasitäre Orientierung }\end{array}$ & Unzureichende Ärgerkontrolle \\
\hline $\begin{array}{l}\text { Erheblich übersteigertes } \\
\text { Selbstwertgefühl }\end{array}$ & Oberflächliche Gefühle & Fehlen von Zielen & Frühe Verhaltensprobleme \\
\hline Pathologisches Lügen & Gefühlskalt/Mangel an Empathie & Impulsivität & $\begin{array}{l}\text { Schwerwiegendes kriminelles Ver- } \\
\text { halten }\end{array}$ \\
\hline $\begin{array}{l}\text { Manipulation zum persönli- } \\
\text { chen Vorteil }\end{array}$ & $\begin{array}{l}\text { Mangelnde Bereitschaft, Verant- } \\
\text { wortung zu übernehmen }\end{array}$ & Verantwortungslosigkeit & Polytrope Kriminalität \\
\hline
\end{tabular}

Verhaltensweisen dient. Diese Dimensionen beinhalten somit v.a. im interpersonalen als auch im affektiven Faktor die ,callous-unemotional traits (CU traits)“, welche als Persönlichkeitsmerkmale bisweilen auch unabhängig von dissozialem Verhalten beobachtet werden können (Moffitt 2018).

Drei- und Vier-Faktoren-Modelle erzielen als Psychopathy-Konstrukte gute Fitwerte (Salekin 2016; Kosson et al. 2013; Jones et al. 2006) und scheinen eine dimensionale Darstellung und detaillierte Untersuchung der einzelnen Komponenten der Konzepte zu ermöglichen (Hare und Neumann 2008; Smith et al. 2009). Dies erlaubt eine differenziertere Sicht auf mögliche Ursachen für die Ausprägung gewisser Psychopathy-Charaktereigenschaften und deren in jedem Individuum anders akzentuierten - Gewichtungen.

\section{Epidemiologie der Psychopathy im Kindes- und Jugendalter}

Obwohl die Psychopathy eine der am besten empirisch validierten Persönlichkeitspathologien darstellt (Miller et al. 2008), bleibt die Konzeptualisierung eines allgemeinen Psychopathy-Konstrukts jedoch weiterhin ein heterogenes Feld, in dem uneinheitliche Messstandards nur bedingt vergleichbare Messwerte erzeugen und einzelne Studien Fragen über die Validität der Drei- und Vier-FaktorenModelle bei weniger beforschten Subgruppen wie Kindern und Jugendlichen aufwerfen (Vitacco et al. 2006).

Des Weiteren erschwert die bisher nicht abschließend beantwortete Frage nach der Definition der Psychopathy, und insbesondere der Psychopathy bei Kindern und Jugendlichen, die epidemiologische Datenerhebung. Bei Kindern und Jugendlichen mangelt es an Daten zur Prävalenz von Psychopathy, da verschiedenen Studien unterschiedliche Instrumente und Schwellenwerte zugrunde liegen und den spezifischen Dimensionen der Psychopathy unterschiedlichste Bedeutung zugeschrieben wird. In der Allgemeinbevölkerung wird von Prävalenzen für Psychopathy von 0,75 bis $1 \%$ ausgegangen (Werner et al. 2015) - ob diese Werte auf Kinder und Jugendliche umzulegen sind, ist unbekannt. In Stichproben jugendlicher Straftäter hängt die Psychopathy-Prävalenz stark von der Gewichtung hoch ausgeprägter Merkmale wie Stimulationsbedürfnis und Impulsivität ab, welche den Schwellenwert der Psychopathy Checklist: Youth Version (PCL:YV) verfälschend erhöhen. Wird in diesen Stichproben eine sehr enge Definition von Psychopathy angewendet, welche adoleszenztypische Verhaltensmerkmale des Lifestyle-Faktors sowie antisoziale Merkmale ausschließt und gleichzeitig hohe Ausprägungen der Kerndimensionen der Psychopathy erfordert, so findet sich dieses Psychopathy-Konstrukt in jugendlichen Delinquenten bei $27 \%$ der Jungen und 7\% der Mädchen (Sevecke et al. 2011).

\section{Neue Modelle und Marker bei Kindern und Jugendlichen mit Psychopathy- Eigenschaften}

Nicht nur die Revision der Psychopathy-Konstrukte, sondern auch das Forschen nach psychophysischen, biologischen, neuronalen und anderen Markern kann helfen, die Ätiologie der Psychopathy besser zu verstehen und rechtzeitig zu intervenieren. Jugendliche mit psychopathischen Eigenschaften weisen z.B. einen geringeren Ruhepuls auf als gesunde Kontrollgruppen (Raine und Glenn 2014), und zeigen bei Präsentation trauriger Stimuli weniger Abweichung des Ruhepulses (de Wied et al. 2012). Ebenso konnte eine positive Korrelation zwischen beeinträchtigten affektiven Werten und gesteigerter Pulsbeschleunigung festgestellt werden (Wang et al. 2012).

Elektromyographische Messungen dokumentierten reduzierte Schreckreflexe auf aversive Stimuli im Vergleich zu gesunden Kontrollgruppen (Kimonis et al. 2017; Syngelaki et al. 2013). Auch der Hautleitwert, gemessen nach Aussetzung von bedrohlichen oder schreckenden Stimuli, bringt Hinweise auf eine Verbindung zwischen verminderter Angst und hohen Psychopathy-Werten. Bei auffälligen Kindern und Jugendlichen erwies sich dieser als im Vergleich zur Norm verringert (Isen et al. 2010; Fung et al. 2005; Fairchild et al. 2010). 
Was Wang und Kollegen durch eine Zwillingsstudie ferner darlegten, war, dass der Hautleitwert im Zusammenhang mit interpersonell-affektiven Faktoren der Psychopathy zu einem relevanten Prozentsatz genetisch bedingt scheint (Wang et al. 2015). Die Möglichkeit neuer genetischer Marker spiegelt sich jedoch nicht nur auf vegetativer, sondern ebenso auf Verhaltens- und molekularer Ebene wider (Viding et al. 2010; Larsson et al. 2006). Gewisse Variationen des Serotonintransportergens können die Ausprägung von Psychopathy-Persönlichkeitseigenschaften in Jugendlichen beeinflussen (Sadeh et al. 2010); ähnliche Ergebnisse sind auch noch im Erwachsenenalter replizierbar und lassen daher Stabilität vermuten (Tielbeek et al. 2016). Wie in einigen Studien eruiert wurde, scheinen Kortisol-Level und die Interaktion des Kortisols mit anderen Hormonen Auswirkungen auf besagte Eigenschaften zu haben und eine Möglichkeit für zukünftige hormonelle Marker zu eröffnen (Johnson et al. 2014; Kimonis et al. 2017; Stoppelbein et al. 2014).

Am intensivsten wird wohl auf neuronaler Ebene versucht, die Ätiologie der Psychopathy zu entschlüsseln, indem die Struktur des Gehirns, die Konnektivität sowie die Funktionalität anhand von MRT-, fMRT- und EEGMessungen eingehend untersucht wurden. Ergebnisse deuten darauf hin, dass Jugendliche zwischen 12 und 20 Jahren mit Psychopathy-Charaktereigenschaften Abnormalitäten in der grauen Materie einiger Gehirnregionen aufweisen (Cope et al. 2014; Ermer et al. 2013; Rogers und De Brito 2016), Nervenleitbahnen zwischen Gehirnregionen können verändert oder gestört sein (Aghajani et al. 2016; Cohn et al. 2016), ebenso die Funktion der Regionen selbst. Beispiele stellen das „default mode network“, die Amygdala oder der ventromediale Präfrontalkortex dar, welche u.a. mit selbstreferenziellem Denken, moralischem Verhalten und Emotionsverarbeitungsprozessen in Verbindung gebracht werden (Thijssen und Kiehl 2017; Pape et al. 2015). Einen interessanten Ausblick auf zukünftige Möglichkeiten bringt die „Proof-of-concept“-Studie an Jugendlichen (im Durchschnitt 17,29 Jahre alt) von Steele und Kollegen, in der erstmals versucht wurde, mittels maschinellem Lernen und moderner neuraler bildgebender Verfahren Gehirnaktivitätsmuster zu finden, die pathognomonisch für die Diagnose Psychopathy bzw. Verhaltensstörung sind und welche infolge möglicherweise die Unterteilung in spezifischere Subgruppen ermöglichen (Anderson et al. 2017). Der erste Schritt zu Untersuchungen mit jüngeren Probanden (14 bis 15 Jahre) ist bereits erfolgt (Zhang et al. 2018).

Es sind trotz vielversprechender Resultate einige Limitationen dieser Studien zu nennen, allen voran die durchmischten, teils widersprüchlichen Ergebnisse, der oftmals mangelhafte Stichprobenumfang, Gender-Bias, die geringe Studiendichte und ungenügende statistische Aussagekraft.

\section{Diagnoseverfahren bei Kindern und Jugendlichen}

Im Bereich der Forensik stehen hoch ausgeprägte Psychopathy-Dimensionen im Zusammenhang mit schweren Gewalttaten, wiederholter Kriminalität sowie schlechten Behandlungsprognosen (Reidy et al. 2013). Aufgrund des stabil ausgeprägten gewalttätigen Verhaltens und der hohen Rückfallquoten bei Tätern mit hoch ausgeprägten Psychopathy-Merkmalen besteht großes Interesse an deren Früherkennung bei Kindern und Jugendlichen, um frühe Interventions- und Behandlungsstrategien zu entwickeln und schwere Verläufe der Psychopathy zu verhindern. Die Anwendung und Erforschung des Psychopathy-Konstrukts im Kindesund Jugendalter sollen weiter dazu dienen, kriminelles Verhalten in der Adoleszenz zu differenzieren und somit in der heterogenen Gruppe adoleszenter Straftäter spezifische Interventionen entwickeln und anwenden zu können (Campbell et al. 2004).

Mittlerweile besteht Konsensus darüber, dass jene Persönlichkeitsmerkmale und Verhaltensweisen, welche das Konstrukt der Psychopathy auszeichnen, sich bereits vor Erreichen des Erwachsenenalters manifestieren (Frick 2009). Studien haben gezeigt, dass Psychopathy-Merkmale bereits im Alter von 4 bis 6 Jahren beobachtet werden können (Assary et al. 2015; Kimonis et al. 2016).

Die Erhebung von Psychopathy-Merkmalen durch geeignete Messinstrumente soll dazu dienen, frühe Auffälligkeiten möglichst zuverlässig zu erfassen, um durch eine korrekte Diagnoseerhebung den Weg zur Entwicklung effektiver Screening- und Interventionsstrategien zu ebnen. Das gängigste Screeningverfahren für Psychopathy-Traits orientiert sich am Hare-Konstrukt und wurde als Hare Psychopathy Checklist: Youth Version für die Anwendung in einer jugendlichen Population adaptiert und validiert (Grisso et al. 2005; Neumann et al. 2006). Da die Psychopathy nicht als Diagnose in kategorialen Diagnosemanualen wie dem DSM-5 oder der ICD-10 enthalten ist, dienen Instrumente wie die PCL:YV dazu, die dimensional variierenden Persönlichkeitseigenschaften des Psychopathy-Konzepts auch bei Heranwachsenden zu erfassen. In 20 Items erfasst diese Checkliste alle Dimensionen der Psychopathy und dient somit als Verfahren, welches in seiner Anwendung zu Erfassung und Konzeptualisierung des Psychopathy-Konstrukts bei Jugendlichen beitragen kann. Im Gegensatz zu Skalen wie der PCL:SV oder der PCL-R, welche im Erwachsenenalter auch zur Diagnoseerfassung angewendet werden können, liefert die PCL:YV keinen „Cut-off“-Wert für klinische Diagnosen. Dies begründet sich durch die Gefahr der Stigmatisierung von Kindern und Jugendlichen, v. a. in Hinsicht auf fehlende Nachweise für die Stabilität von PsychopathyMerkmalen vom Jugend- ins Erwachsenenalter (Archer und Wheeler 2013). Neben der PCL:YV, welche als halbstruktu- 
riertes Interview angewandt wird, stehen Messinstrumente zur Selbstbeurteilung wie der Youth Psychopathic Traits Inventory (YPI) (van Baardewijk et al. 2008) zur Verfügung, welcher bei Jugendlichen ab 12 Jahren in 10 Subskalen Psychopathy-Eigenschaften im Sinne des Drei-Faktoren-Modells erfasst. Auf ähnliche Weise erfasst die Modified Child Psychopathy Scale ( $m C P S$ ) in Anlehnung an die Child Behavior Checklist (CBCL) (Achenbach 2011) PsychopathyDimensionen in Adoleszenten. Ein weiteres Selbstbeurteilungsinstrument, das Psychopathic Personality InventoryRevised (PPI-R) (Lilienfeld et al. 2005) ist für junge Patienten im Alter von 18 bis 28 Jahren validiert und kann somit auch Aufschluss über Psychopathy-Ausprägung im späten Jugendalter geben. Die Gefahr von Selbstbeurteilungsinstrumenten besteht jedoch v. a. in Strafpopulationen darin, dass diese im forensischen Kontext leichter einer bewussten Manipulation des Patienten anheimfallen können, um etwa die Entscheidung des Justizapparats hinsichtlich Zurechnungsfähigkeit zu beeinflussen (Edens et al. 2000).

In jüngeren Kindern können Verfahren wie das Antisocial Process Screening Device (APSD) (Frick und Hare 2001) und die Childhood Psychopathy Scale (CPS) (Lynam 1997) dazu dienen, Dimensionen der Psychopathy durch Fremdbeurteilung, etwa durch Lehrer oder Eltern, ab dem Alter von 6 Jahren zu erfassen. Mit dem Child Problematic Traits Inventory (CPTI) steht ein relatives neues Verfahren zur Verfügung, das mithilfe von Beurteilung durch Lehrer bereits bei Kindern ab 3 Jahren angewendet werden kann und eine kindliche Version des Drei-Faktoren-Modells der psychopathischen Persönlichkeit erfassen soll (Colins et al. 2014). Zur spezifischen Erfassung von gefühls- und emotionslosen Eigenschaften kann das Inventory of CallousUnemotional Traits (ICU) herangezogen werden, das somit in der Abgrenzung zu anderen antisozialen Persönlichkeitstypen dienen kann (Moore et al. 2017). Um die interpersonalen Dimensionen der Psychopathy besser zu erfassen etwa durch die Beurteilung der Interaktion von Interviewer und Patienten - stellt das Interpersonal Measure of Psychopathy $(I M-P)$ ein hilfreiches Instrument dar, welches im Zusammenhang mit jugendspezifischen Verfahren wie der PCL:YV angewendet werden kann (Zolondek et al. 2006).

Während das gängige Psychopathy-Konstrukt und die darauf aufbauenden Verfahren wie die PCL-R v. a. in männlichen Populationen als nachweisbar valide und zuverlässige Verfahren angewendet werden können, mangelt es an Forschungsergebnissen, welche Erkenntnisse über Psychopathy bei Frauen und Mädchen liefern. Aufgrund der steigenden Raten an Gewaltverbrechen, welche von weiblichen Tätern begangen werden, ist die Frage, ob und wie sich die Faktoren des Psychopathy-Konstrukts in Mädchen manifestieren, von besonderer Bedeutung (Odgers et al. 2005). Studien, welche bei inhaftierten Mädchen mit hoch ausgeprägter Psychopathy Zusammenhänge mit depressiven Sympto- men wie internalisierendem und suizidalem Verhalten fanden, könnten darauf hinweisen, dass die Psychopathy in ihrer derzeitigen Konzeptualisierung bei Mädchen kein ausreichend valides Konstrukt darstellt (Sevecke et al. 2009).

\section{Stabilität von Persönlichkeitsstörungen und Psychopathy im Kindes- und Jugendalter}

Da das Konstrukt der Psychopathy als Persönlichkeitsstörung verstanden werden kann, ist sie Teil der kontrovers diskutierten Frage nach Diagnostizierbarkeit von Persönlichkeitsstörungen im Kindes- und Jugendalter und der Abgrenzung zu Adoleszenzkrisen, welche sich durch temporäre Entwicklungsmerkmale kennzeichnen. Nachweise des Vorliegens von Persönlichkeitsstörungen im Jugendalter (Tackett et al. 2009; Kushner et al. 2013; Scott et al. 2009) stehen im Kontrast zu der in Diagnosemanualen proklamierten Stabilität von Persönlichkeitsstörungen, welche diese als tief verwurzelte Verhaltensweisen beschreiben, was jedoch in aktuellen Studien nicht nachgewiesen werden kann. Somit lassen sowohl die ICD-10 als auch das DSM-IV jugendspezifische Modelle und Entwicklungsaspekte von Persönlichkeitsstörungen vermissen, für welche das DSM-5 durch zusätzliche dimensionale Modelle nur teilweise kompensieren kann (Strickland et al. 2013). Die Diagnose einer Persönlichkeitsstörung im Jugendalter wird daher nach wie vor bedauerlicherweise kategorial erfasst. Die damit einhergehende Beschreibung von spezifischen Verhaltensweisen als durchgehend und situationsübergreifend steht im Zusammenhang mit der Zögerlichkeit, Persönlichkeitsstörungen im Kindes- und Jugendalter zu diagnostizieren. Im Kontrast hierzu liefern aktuelle Studien Nachweise für die Instabilität von kategorialen Persönlichkeitsdiagnosen, welche vor dem Erwachsenenalter erfasst wurden (Chanen et al. 2004). Die Literatur zeigt, dass die Symptomlast von Persönlichkeitsstörungen im Adoleszenzalter am höchsten ist und infolge linear abnimmt (Crawford et al. 2005). Auch Studien an erwachsenen Populationen belegen, dass Persönlichkeitsstörungen einen dynamischen Verlauf aufweisen, welcher durch kategoriale Diagnoseverfahren nicht erfasst werden kann.

Im Hinblick auf Psychopathy haben Studien gezeigt, dass dahingehende Eigenschaften und Tendenzen bei Kindern bzw. Jugendlichen bereits in sehr jungen Jahren nachgewiesen werden können (Assary et al. 2015; Kimonis et al. 2016). Aufgrund des Stigmas und der richtungweisenden Implikationen gibt es bis heute rege Debatten ob der Sinnhaftigkeit, Kinder mit Psychopathy zu diagnostizieren (Lynam et al. 2009). Zwar ist es im Hinblick auf antisoziale Verhaltensauffälligkeit Fakt, dass bestimmte Risikofaktoren für Kinder existieren, welche erhöhte Werte und eine damit verbundene Kriminalität im Erwachsenenalter vor- 
aussagen, diese mahnen jedoch zur Vorsicht. Genetische Einflüsse, Erziehungs- und Familienverhältnisse sowie neurokognitive Marker bei Kindern bestärken die These einer soziokulturellen Entwicklungsdynamik, die es erlaubt, etwa antisozial-verhaltensauffällige männliche Jugendliche in zwei grobe Gruppen zu unterteilen: Durch das immer weiter nach hinten geschobene Erreichen der sozialen Reife im Vergleich zur biologischen Reife entstand in den letzten Jahrzehnten eine „Reifungslücke“ (,maturity gap“) bei vielen Jugendlichen, welche im Zuge des Selbstfindungsprozesses zu transientem delinquenten Verhalten führte. In dieser Gruppe stellt das antisoziale Verhalten lediglich ein refraktäres Zustandsbild ohne wesentliche persönlichkeitsgestörte Tendenzen dar. Stark abzugrenzen ist diese Ausprägung von der weitaus kleineren Gruppe an Jugendlichen, die schon von Kindheit an ein auffällig delinquentes Verhalten und hohe antisoziale Werte zeigen, deren Verlauf bis ins Erwachsenenalter stabil bleibt, schwer behandelbar ist und für das Justiz- und Sozialsystem ungleich mehr Kosten verursacht. Da diese beiden Gruppen somit nahezu komplett verschiedene Herangehensweisen - nicht zuletzt auch in deren Behandlung - erfordern, unterstreicht dies einmal mehr die Notwendigkeit von adäquaten Screeningmethoden und Instrumenten für Psychopathy-Dimensionen im Kindesalter (Moffitt 2018).

\section{Komorbiditäten und Abgrenzung zu anderen Störungsbildern bei Kindern und Jugendlichen}

In Anbetracht des diagnostischen Spektrums verschiedener Persönlichkeitsstörungen sowie der unzureichenden Erfassung von Dimensionalität in den gängigen Diagnosemanualen bestehen zahlreiche Komorbiditäten und Schnittstellen zwischen den einzelnen Störungsbildern, welche die korrekte Diagnosestellung v. a. im klinischen Kontext erschweren.

Somit weisen auch die Persönlichkeitsmerkmale der Psychopathy Überschneidungspunkte mit anderen Störungsbildern, insbesondere der antisozialen, der narzisstischen und der histrionischen Persönlichkeitsstörung im Erwachsenenalter, auf (Ogloff 2006). Im Kindes- und Jugendalter werden Psychopathy-Traits als spezifizierende Faktoren von Störungen des Sozialverhaltens gesehen, jedoch nicht als eigenständiges Störungsbild erfasst (Pisano et al. 2017). Im DSM-5 werden dementsprechend Psychopathy-Merkmale als CU traits, oder gefühls- und emotionslose Eigenschaften, berücksichtigt und erfassen als solche fehlende Reue oder Schuldgefühle, Gleichgültigkeit und Fehlen von Empathie, Gleichgültigkeit gegenüber eigenen Leistungen sowie oberflächliche oder defizitäre Affekte. Dementsprechend kann Psychopathy bei Kindern und Jugendlichen als
Extremform aggressiv-dissozialen Verhaltens von anderen Störungsbildern abgegrenzt werden. Uneinigkeit herrscht bislang darüber, woraus sich der Zusammenhang zwischen ADHS im Kindesalter mit antisozialer Persönlichkeit und Kriminalität im Erwachsenenalter ergibt - klar ist jedoch, dass Störungen des Sozialverhaltens - mit Psychopathy als Extremform - in Kindheit und Jugend eine gewisse Komorbidität mit ADHS aufweisen, welche wiederum laut manchen Hypothesen eine erhöhte Vulnerabilität für Psychopathy zur Folge haben könnte (Lynam 1996).

Forschungsergebnisse bei Erwachsenen zeigen einen deutlichen Zusammenhang zwischen Psychopathy und schwerer, wiederholter Kriminalität, gewalttätigem Verhalten und schlechter Behandlungsprognose (Riser und Kosson 2013). Aus der heterogenen Konzeptualisierung der Psychopathy ergibt sich daher die Notwendigkeit, die verschiedenen Faktoren hinsichtlich ihres Zusammenhangs mit Komorbiditäten wie etwa Rückfallkriminalität zu erforschen. Klinische Studien mit inhaftierten Gewalttätern mit Psychopathy verweisen hierbei auf eine Sonderstellung des interpersonalen Faktors - dieser weist eine deutlich schwächere Assoziation mit anderen Störungsbildern wie Substanzabhängigkeit oder antisozialer Persönlichkeitsstörung auf und ist als prädiktiver Faktor für wiederholte Kriminalität deutlich schwächer ausgeprägt als die restlichen drei Faktoren (affektiv - Lifestyle - antisozial). Im Gegensatz dazu weist der antisoziale Faktor eine ausgeprägte Assoziation mit impulsiven und aggressiven Verhaltensweisen auf - es bestehen gar Hinweise darauf, dass dieser Faktor als ein verlässlicherer Prädiktor für Rückfallkriminalität dienen kann als der PCL-R-Gesamt-Score (Wallinius et al. 2012). In der Erfassung von Psychopathy-Faktoren muss daher jedoch auch die Gefahr einer Verfälschung von PsychopathyScores - im Sinne von falsch-hohen Werten aufgrund von schwerem antisozialen Verhalten - beachtet werden.

\section{Therapeutische Interventionen bei Kindern und Jugendlichen}

In Bezug auf therapeutische Interventionen bei Psychopathy-Patienten hat sich in klinisch-psychiatrischen Kreisen ein gewisser Nihilismus breitgemacht, der sich auf Forschungsergebnisse stützt, welche zeigen, dass Therapieversuche oft auf geringe Behandlungsmotivation stoßen und nur unzufriedenstellend geringe Ergebnisse erbringen (Ogloff James et al. 1990). Diese Erkenntnisse, welche sich größtenteils auf Forschung bei Erwachsenen stützen, konnten auch bei Jugendlichen repliziert werden - hier zeigten sich mehr Therapieabbrüche und weniger Therapiefortschritte bei Jugendlichen mit höheren PCL:YV Scores (Spain Sarah et al. 2004). Der allgemeine therapeutische Pessimismus, welcher sich aufgrund der mangelhaften 
Datenlage bei Kindern und Jugendlichen v. a. aus Erfahrungen mit Erwachsenen und deren spärlichem Ansprechen auf Therapie sowie hohen Rückfallquoten unter Gewalttätern mit hoch ausgeprägten Psychopathy-Dimensionen speist, sollte jedoch nicht kritiklos auf Kinder und Jugendliche übertragen werden. In dieser Patientengruppe ist die Datenlage dürftig, liefert jedoch Hinweise darauf, dass spezifische Interventionsstrategien durchaus Erfolg zeigen und somit einer späteren Therapieresistenz sogar entgegenwirken können, da Psychopathy-Dimensionen in Jugendlichen einen prädiktiven Wert für zukünftige Gewaltbereitschaft liefern (Kahn et al. 2013). So zeigte sich etwa, dass Elterntraining in männlichen Kindern zwischen 4 und 8 Jahren eine Reduktion von $\mathrm{CU}$ traits bewirken konnte (Hawes und Dadds 2005). Eine Studie, welche einen verhaltenstherapeutischen Ansatz anwandte, konnte einen Therapieerfolg bei Jungen und Mädchen im Alter von 6 bis 11 Jahren verbuchen, indem CU traits über einen Behandlungszeitraum von 3 Jahren reduziert werden konnten (Kolko und Pardini 2010).

Ein therapeutischer Ansatz, welcher motivationale Techniken, Verhaltenstherapie und Instruktionen für positive Emotionen vereint, führte im Rahmen eines 12-wöchigen Therapieprogramms dazu, dass ein verbessertes Erleben und Erkennen positiver Emotionen die Therapiemotivation und Änderungsbereitschaft erhöhten und so v.a. zu einer Reduktion des interpersonalen Faktors führten die Tendenzen zu manipulativem Verhalten, Lügen und Grandiosität konnten somit durch gezielte therapeutische Intervention reduziert werden (Salekin et al. 2012). In jugendlichen Straftätern konnte außerdem gezeigt werden, dass eine Intensivierung des Therapieprogramms zu deutlich niedrigeren Rückfallquoten führte (Caldwell et al. 2006).

Trotz dieser vielversprechenden Ergebnisse fehlt es nach wie vor an spezifischen Interventionsstrategien für Kinder und Jugendliche mit Psychopathy-Merkmalen. Aufgrund der fehlenden Empathiefähigkeit sowie Defiziten in der Emotionserkennung, erscheint die mentalisierungsbasierte Therapie als vielversprechende Therapiestrategie bei jungen Menschen mit Psychopathy-Charakteristiken. Dieser Therapieansatz soll dazu dienen, innerpsychische Zustände in sich selbst sowie in anderen Menschen vorstellbar und erlebbar zu machen, um Emotionen und Beweggründe für Verhaltensweisen besser verarbeiten zu können. Aussagekräftige Forschungsarbeiten zur Anwendung der MBT-A bei Psychopathy fehlen bislang; das gute Ansprechen bei Jugendlichen mit Persönlichkeitspathologien wie der Borderline-Persönlichkeitsstörung macht die mentalisierungsbasierte Therapie jedoch zu einem interessanten Forschungspunkt (Taubner et al. 2017). Psychodynamische Therapieverfahren wie etwa die übertragungsfokussierte Psychotherapie nach Otto Kernberg werden bisweilen in fo- rensisch-psychiatrischen Patientenpopulationen angewendet, unter der Annahme, dass den abgewehrten Inhalten von Straftätern mit hoch ausgeprägten Psychopathy-Merkmalen Bindungswünsche zugrunde liegen, die in der Therapeuten-Patienten-Beziehung als Basis für psychotherapeutische Bearbeitung dienen können (Lackinger und Berner 2008). Auch hier fehlen jedoch Forschungsergebnisse bei Kindern und Jugendlichen, welche sich spezifisch auf Patienten mit hoch ausgeprägten Psychopathy-Merkmalen beziehen. Zumindest hinsichtlich antisozialer Verhaltensweisen haben sich Therapieprogramme als wirkungsvoll erwiesen, welche einen spezifisch forensisch-deliktorientierten Ansatz verfolgen (Best et al. o.J.).

\section{Conclusio}

Erste Forschungsergebnisse deuten darauf hin, dass Psychopathy nicht nur im Erwachsenen-, sondern auch im Kindes- und Jugendalter eine schwerwiegende Persönlichkeitspathologie darstellt. Die Hinweise auf das Auftreten von Psychopathy-Merkmalen bereits im frühen Kindheitsalter sowie deren Verlauf im Jugendalter deuten darauf hin, dass eine Früherkennung dieser Erkrankung von größter Bedeutung ist, da hohe Ausprägungen von Psychopathy-Traits zu schwerer, wiederholter Gewalttätigkeit führen können und in späterem Alter nach ersten Erkenntnissen oftmals in therapieresistenter Form auftreten.

Aussagekräftige Forschungsarbeiten über Pathogenese, Gewichtung der einzelnen Dimensionen, Subtypen und Resilienzfaktoren der Psychopathy bei Kindern und Jugendlichen sind rar. Eine gründliche Erforschung dieser Themengebiete ist jedoch unbedingt notwendig, um in einem ersten Schritt die dimensionale, umfassende Diagnostizierung und Subklassifizierung der Psychopathy in jungen Jahren zu ermöglichen. Darauf aufbauend können in einem weiteren Schritt individualisierte Therapiestrategien entwickelt werden, um die betroffenen Kinder und Jugendlichen nach empirischen Gesichtspunkten optimal zu behandeln.

Funding Open access funding provided by University of Innsbruck and Medical University of Innsbruck.

Interessenkonflikt N. Ortner, M. Preiß und K. Sevecke geben an, dass kein Interessenkonflikt besteht.

Open Access Dieser Artikel wird unter der Creative Commons Namensnennung 4.0 International Lizenz (http://creativecommons.org/ licenses/by/4.0/deed.de) veröffentlicht, welche die Nutzung, Vervielfältigung, Bearbeitung, Verbreitung und Wiedergabe in jeglichem Medium und Format erlaubt, sofern Sie den/die ursprünglichen Autor(en) und die Quelle ordnungsgemäß nennen, einen Link zur Creative Commons Lizenz beifügen und angeben, ob Änderungen vorgenommen wurden. 


\section{Literatur}

Achenbach TM (2011) Child behavior checklist. In: Kreutzer JS, DeLuca J, Caplan B (Hrsg) Encyclopedia of clinical neuropsychology. Springer, New York, S 546-552 https://doi.org/10.1007/9780-387-79948-3_1529

Aghajani M, Colins OF, Klapwijk ET, Veer IM, Andershed H, Popma A, van der Wee NJ, Vermeiren RRJM (2016) Dissociable relations between amygdala subregional networks and psychopathy trait dimensions in conduct-disordered juvenile offenders. Hum Brain Mapp 37(11):4017-4033. https://doi.org/10.1002/hbm.23292

Anderson NE, Steele VR, Maurer JM, Rao V, Koenigs MR, Decety J, Kosson DS, Calhoun VD, Kiehl KA (2017) Differentiating emotional processing and attention in psychopathy with functional neuroimaging. Cogn Affect Behav Neurosci 17(3):491-515. https://doi.org/10.3758/s13415-016-0493-5

Archer RP, Wheeler EMA (2013) Forensic uses of clinical assessment instruments. Taylor \& Francis, Abingdon

Assary E, Salekin RT, Barker ED (2015) Big-five and callous-unemotional traits in preschoolers. $J$ Psychopathol Behav Assess 37(3):371-379. https://doi.org/10.1007/s10862-0149471-9

van Baardewijk Y, Stegge H, Andershed H, Thomaes S, Scholte E, Vermeiren R (2008) Measuring psychopathic traits in children through self-report. The development of the youth psychopathic traits inventory-child version. Int J Law Psychiatry 31(3):199-209. https://doi.org/10.1016/j.ijlp.2008.04.004

Best T, Aebi M, Bessler C (2015) Forensisches Therapieprogramm für junge Straftäter: Das ForTiS-Manual. Therapeutische Praxis. Hogrefe, Göttingen

Caldwell M, Skeem J, Salekin R, Van Rybroek G (2006) Treatment response of adolescent offenders with psychopathy features: a 2-year follow-up. Crim Justice Behav 33(5):571-596. https://doi.org/ 10.1177/0093854806288176

Campbell MA, Porter S, Santor D (2004) Psychopathic traits in adolescent offenders: an evaluation of criminal history, clinical, and psychosocial correlates. Behav Sci Law 22(1):23-47. https://doi. org/10.1002/bsl.572

Chanen AM, Jackson HJ, McGorry PD, Allot KA, Clarkson V, Yuen HP (2004) Two-year stability of personality disorder in older adolescent outpatients. J Pers Disord 18(6):526-541. https://doi.org/ 10.1521/pedi.18.6.526.54798

Cleckley HM (1941) The mask of sanity; an attempt to reinterpret the so-called psychopathic personality. Mosby, Maryland Heights, St. Louis

Cohn MD, van Lith K, Kindt M, Pape LE, Doreleijers TA, van den Brink W, Veltman DJ, Popma A (2016) Fear extinction, persistent disruptive behavior and psychopathic traits: fMRI in late adolescence. Soc Cogn Affect Neurosci 11(7):1027-1035. https://doi. org/10.1093/scan/nsv067

Colins OF, Andershed H, Frogner L, Lopez-Romero L, Veen V, Andershed A-K (2014) A new measure to assess psychopathic personality in children: the child problematic traits inventory. J Psychopathol Behav Assess 36(1):4-21. https://doi.org/10. 1007/s10862-013-9385-y

Cooke DJ, Michie C (2001) Refining the construct of psychopathy: towards a hierarchical model. Psychol Assess 13(2):171-188

Cooke DJ, Hart SD, Logan C, Michie C (2012) Explicating the construct of psychopathy: development and validation of a conceptual model, the comprehensive assessment of psychopathic personality (CAPP). Int J Forensic Ment Health 11(4):242-252. https://doi. org/10.1080/14999013.2012.746759

Cope LM, Ermer E, Nyalakanti PK, Calhoun VD, Kiehl KA (2014) Paralimbic Gray matter reductions in incarcerated adolescent females with psychopathic traits. J Abnorm Child Psychol 42(4):659-668. https://doi.org/10.1007/s10802-013-9810-4
Crawford TN, Cohen P, Johnson JG, Kasen S, First MB, Gordon K, Brook JS (2005) Self-reported personality disorder in the children in the community sample: convergent and prospective validity in late adolescence and adulthood. J Pers Disord 19(1):30-52. https://doi.org/10.1521/pedi.19.1.30.62179

Edens JF, Buffington JK, Tomicic TL (2000) An investigation of the relationship between psychopathic traits and malingering on the psychopathic personality inventory. Assessment 7(3):281-296. https://doi.org/10.1177/107319110000700307

Ermer E, Cope LM, Nyalakanti PK, Calhoun VD, Kiehl KA (2013) Aberrant Paralimbic Gray matter in incarcerated Male adolescents with psychopathic traits RH: Paralimbic Gray matter and psychopathy. J Am Acad Child Adolesc Psychiatry 52(1):94-103. https://doi.org/10.1016/j.jaac.2012.10.013

Fairchild G, Stobbe Y, van Goozen SH, Calder AJ, Goodyer IM (2010) Facial expression recognition, fear conditioning, and startle modulation in female subjects with conduct disorder. Biol Psychiatry 68(3):272-279. https://doi.org/10.1016/j.biopsych.2010.02.019

Frick PJ (2009) Extending the construct of psychopathy to youth: implications for understanding, diagnosing, and treating antisocial children and adolescents. Can J Psychiatry 54(12):803-812. https://doi.org/10.1177/070674370905401203

Frick PJ, Hare RD (2001) Antisocial process screening device: APSD. Multi-Health Systems, Toronto

Fung MT, Raine A, Loeber R, Lynam DR, Steinhauer SR, Venables PH, Stouthamer-Loeber M (2005) Reduced electrodermal activity in psychopathy-prone adolescents. J Abnorm Psychol 114(2):187-196. https://doi.org/10.1037/0021-843x.114.2.187

Grisso T, Vincent G, Seagrave D (2005) Mental health screening and assessment in juvenile justice. Guilford, New York

Hare RD (2003) Hare Psychopathy Checklist-revised (PCL-R): Technical Manual, 2. Aufl. Multi-Health Systems, Toronto

Hare RD, Neumann CS (2008) Psychopathy as a clinical and empirical construct. Annu Rev Clin Psychol 4:217-246. https://doi.org/10. 1146/annurev.clinpsy.3.022806.091452

Hare RD, Hart SD, Harpur TJ (1991) Psychopathy and the DSMIV criteria for antisocial personality disorder. J Abnorm Psychol 100(3):391-398

Harpur TJ, Hare RD, Hakstian AR (1989) Two-factor conceptualization of psychopathy: Construct validity and assessment implications. Psychol Assess 1(1):6-17

Hawes DJ, Dadds MR (2005) The treatment of conduct problems in children with callous-unemotional traits. J Consult Clin Psychol 73(4):737-741. https://doi.org/10.1037/0022-006x.73.4.737

Isen J, Raine A, Baker L, Dawson M, Bezdjian S, Lozano DI (2010) Sex-specific association between psychopathic traits and electrodermal reactivity in children. J Abnorm Psychol 119(1):216-225. https://doi.org/10.1037/a0017777

Johnson MM, Caron KM, Mikolajewski AJ, Shirtcliff EA, Eckel LA, Taylor J (2014) Psychopathic traits, empathy, and aggression are differentially related to cortisol awakening response. J Psychopathol Behav Assess 36(3):380-388

Jones S, Cauffman E, Miller JD, Mulvey E (2006) Investigating different factor structures of the psychopathy checklist: youth version: confirmatory factor analytic findings. Psychol Assess 18(1):33-48. https://doi.org/10.1037/1040-3590.18.1.33

Kahn RE, Byrd AL, Pardini DA (2013) Callous-unemotional traits robustly predict future criminal offending in young men. Law Hum Behav 37(2). https://doi.org/10.1037/b0000003

Kimonis ER, Fanti KA, Anastassiou-Hadjicharalambous X, Mertan B, Goulter N, Katsimicha E (2016) Can callous-unemotional traits be reliably measured in preschoolers? J Abnorm Child Psychol 44(4):625-638. https://doi.org/10.1007/s10802-015-0075-y

Kimonis ER, Fanti KA, Goulter N, Hall J (2017) Affective startle potentiation differentiates primary and secondary variants of juvenile psychopathy. Dev Psychopathol 29(4):1149-1160. https://doi. org/10.1017/s0954579416001206 
Kolko DJ, Pardini DA (2010) ODD dimensions, ADHD, and callous-unemotional traits as predictors of treatment response in children with disruptive behavior disorders. J Abnorm Psychol 119(4):713-725. https://doi.org/10.1037/a0020910

Kosson DS, Neumann CS, Forth AE, Salekin RT, Hare RD, Krischer MK, Sevecke K (2013) Factor structure of the hare psychopathy checklist: youth version (PCL:YV) in adolescent females. Psychol Assess 25(1):71-83. https://doi.org/10.1037/a0028986

Kushner SC, Tackett JL, De Clercq B (2013) The joint hierarchical structure of adolescent personality pathology: converging evidence from two approaches to measurement. J Can Acad Child Adolesc Psychiatry 22(3):199-205

Lackinger F, Berner W (2008) Psychodynamische Psychotherapie bei Delinquenz: Praxis der übertragungsfokussierten Psychotherapie. Schattauer, Stuttgart

Larsson H, Andershed H, Lichtenstein P (2006) A genetic factor explains most of the variation in the psychopathic personality. J Abnorm Psychol 115(2):221-230. https://doi.org/10.1037/ 0021-843x.115.2.221

Lilienfeld SO, Widows MR, Staff PAR (2005) Psychopathic personality inventoryTM-revised. Soc Influ 61(65):97

Lykken DT (1957) A study of anxiety in the sociopathic personality. J Abnorm Psychol 55(1):6-10

Lynam DR (1996) Early identification of chronic offenders: who is the fledgling psychopath? Psychol Bull 120(2):209-234

Lynam DR (1997) Pursuing the psychopath: Capturing the fledgling psychopath in a nomological net. J Abnorm Psychol 106(3):425

Lynam DR, Charnigo R, Moffitt TE, Raine A, Loeber R, Stouthamer-Loeber M (2009) The stability of psychopathy across adolescence. Dev Psychopathol 21(4):1133-1153. https://doi.org/10. 1017/S0954579409990083

Miller JD, Gaughan ET, Pryor LR (2008) The Levenson Self-Report Psychopathy scale: an examination of the personality traits and disorders associated with the LSRP factors. Assessment 15(4):450-463. https://doi.org/10.1177/1073191108316888

Moffitt TE (2018) Male antisocial behaviour in adolescence and beyond. Nat Hum Behav 2(3):177-186

Moore AA, Carney D, Moroney E, Machlin L, Towbin KE, Brotman MA, Pine DS, Leibenluft E, Roberson-Nay R, Hettema JM (2017) The inventory of callous-unemotional traits (ICU) in children: reliability and heritability. Behav Genet 47(2):141-151. https://doi. org/10.1007/s10519-016-9831-1

Neumann CS, Kosson DS, Forth AE, Hare RD (2006) Factor structure of the Hare psychopathy checklist: youth version (PCL: YV) in incarcerated adolescents. Psychol Assess 18(2):142-154. https:// doi.org/10.1037/1040-3590.18.2.142

Odgers CL, Reppucci ND, Moretti MM (2005) Nipping psychopathy in the bud: an examination of the convergent, predictive, and theoretical utility of the PCL-YV among adolescent girls. Behav Sci Law 23(6):743-763. https://doi.org/10.1002/bsl.664

Ogloff JR (2006) Psychopathy/antisocial personality disorder conundrum. Aust N Z J Psychiatry 40(6-7):519-528. https://doi.org/10. 1080/j.1440-1614.2006.01834.x

Ogloff James RP, Wong S, Greenwood A (1990) Treating criminal psychopaths in a therapeutic community program. Behav Sci Law 8(2):181-190. https://doi.org/10.1002/bs1.2370080210

Pape LE, Cohn MD, Caan MW, van Wingen G, van den Brink W, Veltman DJ, Popma A (2015) Psychopathic traits in adolescents are associated with higher structural connectivity. Psychiatry Res 233(3):474-480. https://doi.org/10.1016/j.pscychresns.2015.07. 023

Patrick CJ (2018) Handbook of psychopathy, 2. Aufl. Guilford, New York

Pisano S, Muratori P, Gorga C, Levantini V, Iuliano R, Catone G, Coppola G, Milone A, Masi G (2017) Conduct disorders and psychopathy in children and adolescents: aetiology, clinical presentation and treatment strategies of callous-unemotional traits. Ital J Pediatr 43. https://doi.org/10.1186/s13052-017-0404-6
Raine A, Glenn AL (2014) Psychopathy: an introduction to biological findings and their implications. Psychology and crime. NYU Press, New York

Reidy DE, Kearns MC, DeGue S (2013) Reducing psychopathic violence: A review of the treatment literature. Aggress Violent Behav 18(5):527-538. https://doi.org/10.1016/j.avb.2013.07.008

Riser RE, Kosson DS (2013) Criminal behavior and cognitive processing in male offenders with antisocial personality disorder with and without comorbid psychopathy. Personal Disord 4(4):332-340. https://doi.org/10.1037/a0033303

Rogers JC, De Brito SA (2016) Cortical and Subcortical Gray matter volume in youths with conduct problems: a Meta-analysis. JAMA Psychiatry 73(1):64-72. https://doi.org/10.1001/jamapsychiatry. 2015.2423

Sadeh N, Javdani S, Jackson JJ, Reynolds EK, Potenza MN, Gelernter J, Lejuez CW, Verona E (2010) Serotonin transporter gene associations with psychopathic traits in youth vary as a function of socioeconomic resources. J Abnorm Psychol 119(3):604-609. https://doi.org/10.1037/a0019709

Salekin RT (2016) Psychopathy in childhood: Toward better informing the DSM-5 and ICD-11 conduct disorder specifiers. Personal Disord 7(2):180-191. https://doi.org/10.1037/per0000150

Salekin RT, Tippey JG, Allen AD (2012) Treatment of conduct problem youth with interpersonal callous traits using mental models: measurement of risk and change. Behav Sci Law 30(4):470-486. https://doi.org/10.1002/bsl.2025

Scott LN, Levy KN, Pincus AL (2009) Adult attachment, personality traits, and borderline personality disorder features in young adults. J Pers Disord 23(3):258-280. https://doi.org/10.1521/pedi. 2009.23.3.258

Sevecke K, Krischer MK (2014) PCL:YV hare psychopathy checklist: youth version by A.E. Forth, D.S. Kosson and R.D. Hare. Hogrefe, Göttingen (Deutsche Version)

Sevecke K, Lehmkuhl G, Krischer MK (2009) Examining relations between psychopathology and psychopathy dimensions among adolescent female and male offenders. Eur Child Adolesc Psychiatry 18(2):85-95. https://doi.org/10.1007/s00787-008-0707-7

Sevecke K, Lehmkuhl G, Krischer MK (2011) Epidemiological data on personality dimensions in the psychopathy of adolescent boys and girls-results of the Cologne GAP study. Z Kinder Jugendpsychiatr Psychother 39(1):9-20. https://doi.org/10.1024/14224917/a000079 (quiz 20-21)

Smith GT, McCarthy DM, Zapolski TC (2009) On the value of homogeneous constructs for construct validation, theory testing, and the description of psychopathology. Psychol Assess 21(3):272-284. https://doi.org/10.1037/a0016699

Spain SE, Douglas KS, Poythress NG, Epstein M (2004) The relationship between psychopathic features, violence and treatment outcome: the comparison of three youth measures of psychopathic features. Behav Sci Law 22(1):85-102. https://doi.org/10.1002/ bsl.576

Stoppelbein L, Greening L, Luebbe A, Fite P, Becker SP (2014) The role of cortisol and psychopathic traits in aggression among at-risk girls: tests of mediating hypotheses. Aggress Behav 40(3):263-272. https://doi.org/10.1002/ab.21513

Strickland CM, Drislane LE, Lucy M, Krueger RF, Patrick CJ (2013) Characterizing psychopathy using DSM-5 personality traits. Assessment 20(3):327-338. https://doi.org/10.1177/ 1073191113486691

Syngelaki EM, Fairchild G, Moore SC, Savage JC, van Goozen SH (2013) Fearlessness in juvenile offenders is associated with offending rate. Dev Sci 16(1):84-90. https://doi.org/10.1111/j.14677687.2012.01191.x

Tackett JL, Balsis S, Oltmanns TF, Krueger RF (2009) A unifying perspective on personality pathology across the life span: developmental considerations for the fifth edition of the diagnostic 
and statistical manual of mental disorders. Dev Psychopathol 21(3):687-713. https://doi.org/10.1017/s095457940900039x

Taubner S, Volkert J, Gablonski TC, Rossouw T (2017) Mentalizationbased treatment for adolescents with borderline personality disorder-concept and efficacy. Prax Kinderpsychol Kinderpsychiatr 66(6):423-434. https://doi.org/10.13109/prkk.2017.66.6.423

Thijssen S, Kiehl KA (2017) Functional connectivity in incarcerated male adolescents with psychopathic traits. Psychiatry Res Neuroimaging 265:35-44. https://doi.org/10.1016/j.pscychresns.2017. 05.005

Tielbeek JJ, Karlsson Linner R, Beers K, Posthuma D, Popma A, Polderman TJ (2016) Meta-analysis of the serotonin transporter promoter variant (5-HTTLPR) in relation to adverse environment and antisocial behavior. Am J Med Genet B Neuropsychiatr Genet 171(5):748-760. https://doi.org/10.1002/ajmg.b.32442

Viding E, Hanscombe KB, Curtis CJ, Davis OS, Meaburn EL, Plomin $R$ (2010) In search of genes associated with risk for psychopathic tendencies in children: a two-stage genome-wide association study of pooled DNA. J Child Psychol Psychiatry 51(7):780-788. https://doi.org/10.1111/j.1469-7610.2010.02236.x

Vitacco MJ, Neumann CS, Caldwell MF, Leistico AM, Van Rybroek GJ (2006) Testing factor models of the psychopathy checklist: youth version and their association with instrumental aggression. J Pers Assess 87(1):74-83. https://doi.org/10.1207/ s15327752jpa8701_06

Wallinius M, Nilsson T, Hofvander B, Anckarsater H, Stalenheim G (2012) Facets of psychopathy among mentally disordered offenders: clinical comorbidity patterns and prediction of violent and criminal behavior. Psychiatry Res 198(2):279-284. https://doi. org/10.1016/j.psychres.2012.01.005

Wang P, Baker LA, Gao Y, Raine A, Lozano DI (2012) Psychopathic traits and physiological responses to aversive stimuli in children aged 9-11 years. J Abnorm Child Psychol 40(5):759-769. https:// doi.org/10.1007/s10802-011-9606-3

Wang P, Gao Y, Isen J, Tuvblad C, Raine A, Baker LA (2015) Genetic covariance between psychopathic traits and anticipatory skin conductance responses to threat: Evidence for a potential endophenotype. Dev Psychopathol 27(4):1313-1322. https://doi.org/ 10.1017/s0954579414001424

Werner KB, Few LR, Bucholz KK (2015) Epidemiology, comorbidity, and behavioral genetics of antisocial personality disorder and psychopathy. Psychiatr Ann 45(4):195-199. https://doi.org/10.3928/ 00485713-20150401-08

de Wied M, van Boxtel A, Matthys W, Meeus W (2012) Verbal, facial and autonomic responses to empathy-eliciting film clips by disruptive male adolescents with high versus low callous-unemotional traits. J Abnorm Child Psychol 40(2):211-223. https://doi. org/10.1007/s10802-011-9557-8

Zhang J, Liu W, Zhang J, Wu Q, Gao Y, Jiang Y, Gao J, Yao S, Huang B (2018) Distinguishing adolescents with conduct disorder from typically developing youngsters based on pattern classification of brain structural MRI. Front Hum Neurosci 12:152

Zolondek S, Lilienfeld SO, Patrick CJ, Fowler KA (2006) The interpersonal measure of psychopathy: construct and incremental validity in Male prisoners. Assessment 13(4):470-482. https://doi.org/10. $1177 / 1073191106289861$ 